Pacific Journal of Mathematics

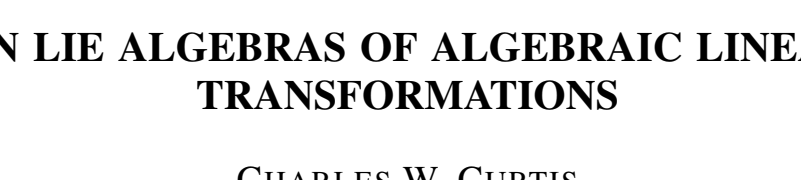




\title{
ON LIE ALGEBRAS OF ALGEBRAIC LINEAR TRANSFORMATIONS
}

\author{
Charles W. Curtis
}

1. Introduction. This paper is concerned with the structure of locally finite Lie algebras of algebraic linear transformations in an infinite dimensional space. The main results include generalizations of the Cartan-Jacobson theorem [7] on completely reducible Lie algebras of linear transformations, Lie's theorem [3] on irreducible representations of solvable Lie algebras, and Malcev's theorem [14] on the structure of splittable algebras. The methods of proof are essentially the known ones, except than in each case suitable reductions to finite dimensional situations must be found.

We obtain the following two criteria in order that a Lie algebra $\&$ of algebraic linear transformations be commutative:

(A) $\mathfrak{R}$ is solvable, and the enveloping associative algebra $\mathfrak{A}$ of $\mathbb{R}$ is semi-simple in the sense of Jacobson [9];

(B) $\mathfrak{L}$ is solvable, of countable dimension over an algebraically closed field, and the linear transformations in $\&$ are semi-simple. An example shows that in neither case need all the row-finite matrices corresponding to the elements of $\mathfrak{R}$, relative to a fixed basis of the vector space, be diagonal, contrary to the situation which prevails in the finite dimensional case.

We summarize briefly the contents of the paper. It is proved first, in greater generality than is needed for this paper, that a locally algebraic linear transformation $A$ can be expressed uniquely as a sum $A=S+N$ of a semi-simple locally algebraic linear transformation $S$ and a locally nilpotent transformation $N$, both of which commute which $A$, and can be approximated by polynomials in $A$. In $\S 3$ it is proved that a locally finite Lie algebra $\mathfrak{Q}$ of algebraic linear transformations whose enveloping associative algebra is semi-simple, is a direct sum of an ideal $\mathfrak{L}_{1}$ containing [ $\left.\mathbb{R}, 2\right]$, and the center $\mathfrak{c}$; moreover $\mathfrak{L}_{1}$ contains no nonzero solvable ideals, and every element of $\sqrt{ } \sqrt{ }$ is semi-simple. An example is given which indicates that $[\Omega, \Omega]$ may be properly contained in $\Omega_{1}$. In $\S 4$, Theorem (A) is proved, together with the fact that any solvable Lie algebra of algebraic linear transformations is locally finite. The final section contains a discussion of linearly splittable algebras, the generalization of Malcev's theorem, Theorem (B), and an example which shows that the countability hypotheses which are introduced in the section cannot be removed.

Received March 14, 1955. Part of this work was done while the author was a National Research Fellow, Presented to the American Mathematical Society October 30, 1954. 
We remark that a number of results concerning infinite dimensional nilpotent Lie algebras, and other theorems related to the subject of this paper have been obtained by Schenkman [15], and by Drazin and Gruenberg [4], [5].

Thanks are due to the referee, who suggested simplifications in the proofs of Theorems 2.1 and 2.3 , and an improvement in $\S 5$.

2. Preliminary results on locally algebraic linear transformations. Let $\mathfrak{M}$ be a vector space over a perfect field $K$. If $\subseteq$ is a set of linear transformations (l.t.) acting in $\mathfrak{M}$, then the pair $(\mathfrak{M}$, $\subseteq$ ) is called an S-module. A subspace $\mathfrak{N}$ of $\mathfrak{M}$ which is invariant relative to the l.t. in $\mathfrak{S}$ will be called a submodule; $\mathfrak{N}$ is irreducible if the only submodules

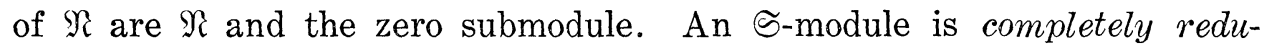
cible if any one of the following three equivalent conditions is satisfied:

(i) $\mathfrak{M}$ is a sum (not necessarily direct) of irreducible submodules;

(ii) $\mathfrak{M}$ is a direct sum of irreducible submodules;

(iii) if $\mathfrak{R}$ is a submodule, then there exists a submodule $\mathfrak{N}^{\prime}$ such that $\mathfrak{M}=\mathfrak{N} \oplus \mathfrak{N}^{\prime} . \quad$ A l.t. is semi-simple if the module determined by this 1.t. is completely reducible. A l.t. $A$ is locally algebraic if every vector $x \in \mathfrak{M}$ is contained in a finite dimensional submodule; $A$ is algebraic if $f(A)=0$, where $f$ is a polynomical with coefficients in $K$; and $A$ is locally nilpotent if $x \in \mathfrak{M}$ implies $x A^{t}=0$, for some $t$ depending upon $x$.

We shall assume the finite dimensional cases of the following theorems; for their proofs see for example Chevalley [1].

Theorem 2.1. Let $A$ be a locally algebraic l.t. in a vector space over a perfect field $K$. Then there exist a semi-simple locally algebraic l.t. $S$ and a locally nilpotent l.t. $N$, both commuting with $A$, such that $A=S+N$. The l.t. $S$ and $N$ are uniquely determined by these properties. If $\widetilde{F}$ is any finite dimensional A-submodule, then there exist polynomials $f_{1}$ and $f_{2}$ in $K[t]$, depending upon $\mathfrak{F}$, such that $f_{1}\left(A_{F^{\prime}}\right)=S_{F}$, and $f_{:}\left(A_{F}\right)=N_{F}$, where $A_{F}, S_{F}$, and $N_{F}$ denote the restrictions of $A, S$, and $N$ to $\mathfrak{F}$.

Proof. Let $\mathfrak{F}$ be a finite dimensional $A$-submodule of $\mathfrak{M i}$ and let $A_{F}$ be the restriction of $A$ to $₹$. By [1, Theorem 2], we can express $A_{F}=S_{F}+N_{F}$, where the summands are uniquely determined polynomials in $A_{F}$ such that $S_{F}$ is semi-simple and $N_{F}$ is nilpotent. Define $S$ and $N$ by $x S=x S_{F}, x N=x N_{F}$, whenever $x \in \mathfrak{F}$. Since every $x$ is contained in an $\mathfrak{F}$, the domain of $S$ and $N$ is all of $\mathfrak{M}$. Since any two finite dimensional submodules are contained in a single one $\mathfrak{F}$, and since the decomposition $A_{F}=S_{F}+N_{F}$ is unique on $\mathfrak{F}$, it follows that $S$ and $N$ are single valued. Clearly these are semi-simple and locally nilpotent, respectively. Now let $A=Y+Z$ be another decomposition of $A$ as a sum 
of a locally nilpotent l.t. $Z$ and a semi-simple locally algebraic l.t. $Y$, where both $Y$ and $Z$ commute with $A$. Then $Y$ and $Z$ commute with $S$ and $N$ because of the way $S$ and $N$ were defined. If $Y$ and $Z$ commute, then since all are locally algebraic, any $x$ is contained in a finite dimensional subspace invariant under $S, N, Y$, and $Z$. Then $S_{F}=Y_{F}, N_{F}=Z_{F}$ by the finite dimensional case. The uniqueness result holds even if it is not assumed that $Y$ and $Z$ commute with each other; in this case we prove that $U=S-Y=Z-N$ is both locally nilpotent and semi-simple. Clearly $U=Z-N$ is locally nilpotent. Any $x$ in $\mathfrak{M}$ is contained in a finite dimensional space $\widetilde{F}$ invariant under $S$ and $Y$. Since the algebra $K\left[1, S_{F}, Y_{F}\right]$ generated by these restrictions is a homomorphic image of the Kronecker product of the finite dimensional semi-simple algebras $K\left[1, S_{F}\right]$ and $K\left[1, Y_{F}\right]$ over the perfect field $K$, it follows that $K\left[1, S_{F}, Y_{F}\right]$, and hence $K\left[1, S_{F}-Y_{F}\right]$ are semi-simple. Then $S_{F}-Y_{F}$ is a semi-simple 1.t., and it follows that $U$ is semi-simple. Then as in [1, Theorem 2], we infer that $U=0$, and the uniqueness is proved. The third statement is clear by the manner in which $S$ and $N$ were constructed.

We shall call $S$ and $N$ the semi-simple and (locally) nilpotent components of $A$.

THEOREM 2.2. If $A$ is an algebraic l.t. then the semi-simple and locally nilpotent components of $A$ are algebraic and nilpotent respectively. and can be expressed as polynomials in $A$.

Proof. This result is an immediate consequence of Chevalley's proof of the theorem in the finite dimensional case and the uniqueness statement of Theorem 2.1.

It should be mentioned that Theorem 2.2 is a special case of the Wedderburn principal theorem, applied to the algebra $K[1, A]$, while the uniqueness, at least when $Y$ and $Z$ commute, is a consequence of Malcev's theorem, applied to the commutative algebra $K[1, S, N, Y, Z]$.

If $S$ is a l.t. let adS denote the mapping $X \rightarrow[X S]$ in the set $[\xi$ of all 1.t. of $\mathfrak{M}$; if $S$ is an element of a Lie subalgebra $\mathfrak{R}$ of $\mathfrak{F}$, then we shall write $a d_{I} S$ for the restriction of $a d S$ to $\mathbb{2}$. when there is no possibility of confusion, we shall write adS for $a d_{L} S$.

TheOREM 2.3. If $A$ is an algebraic l.t. in a Lie algebra \& of l.t., and if $A=S+N$ is the decomposition of $A$ into its semi-simple and nilpotent components, then the mappings $a d_{L} A, a d_{L} S$ and $a d_{L} N$ are algebraic l.t. sending $\mathfrak{Q} \rightarrow \mathfrak{R}$, and $a d_{L} S$ and $a d_{L} N$ are the semi-simple and nilpotent components of $a d_{I} A$. 
Proof. In 5 we have $a d A=a d S+a d N$, where $a d S$ and $a d N$ both commute with $a d A$. Let us denote the commuting right and left multiplications in $\&$ determined by a l.t. $X$ by $R_{X}$ and $L_{X}$ respectively. Then we have $a d N=R_{N}-L_{N}, a d S=R_{S}-L_{S}$. Clearly $a d N$ is nilpotent. Chevalley's construction of $S$ in the finite dimensional case shows that $S$ satisfied a polynomial equation where the polynomial has distinct irreducible factors. Both $R_{S}$ and $L_{S}$ satisfy the same equations, and hence, as in the finite dimensional case, $R_{S}$ and $L_{S}$ are semi-simple. It follows that $a d S$ is semi-simple and algebraic. Then $a d A$ is algebraic, and the uniqueness assertion of Theorem 2.1 implies that adS and adN are the semi-simple and nilpotent components of $a d A$. Since they are both polynomials in $a d A$, they map $\mathbb{2}$ into $\mathfrak{\Omega}$, and it follows that their restrictions to $\mathfrak{Z}$ are the semi-simple and nilpotent components of $a d_{L} A$.

\section{A generalization of a theorem of E. Cartan and N. Jacobson.} The result we shall generalize asserts that a completely reducible Lie algebra of 1.t. in a finite dimensional space over a field of characteristic zero is a direct sum of its derived algebra and its center, the derived algebra is semi-simple, and every l.t. in the center is semi-simple.

THeOREm 3.1. Let \& be a locally finite Lie algebra of algebraic l.t, acting in a vector space $\mathfrak{M}$ over an arbitrary field $K$ of characteristic zero. If the enveloping associative algebra of $\mathbb{a}$ contains no nonzero nil two-sided ideals ${ }^{2}$, then $\mathfrak{L}=\mathfrak{R}_{1} \oplus \mathfrak{S}$, where $\mathfrak{S}$ is the center of $\mathfrak{R}$, and $\mathfrak{R}_{1}$ is an ideal in $\mathfrak{R}$ containing [R, $\mathbb{R}]$ and possessing no nonzero solvable ideals. Furthermore, every l.t. in 5 is semi-simple.

Before proving the theorem, we establish a lemma.

Lemma 3.1. Let $\mathbb{\Omega}$ be a Lie algebra of algebraic l.t. in a vector space $\mathfrak{M}$ over $K$, and let $\mathfrak{A}$ be the enveloping algebra of $\mathbb{2}$. Then $\mathbb{Z}$ is locally finite if and only if $\mathfrak{A}$ is locally finite ${ }^{3}$.

Proof. Let $\Omega$ be locally finite, and let $A_{1}, \cdots, A_{m}$ be a finite set of elements of $\mathfrak{A}$. Then all the $A_{i}$ are in the enveloping algebra $\widetilde{5}$ of a subalgebra $\mathfrak{\Omega}_{1}$ of $\mathfrak{\Omega}$ generated by a finite set $X_{1}, \cdots, X_{p}$ of l.t.. Since $\mathfrak{\Omega}$ is locally finite, $\mathfrak{\Omega}_{1}$ is finite dimensional. Let $Y_{1}, \cdots, Y_{q}$ be a basis for $\mathfrak{R}_{1}$. By the well-known straightening process, it follows that the elements of $\mathfrak{F}$, and in particular the $A_{i}$, can be expressed as linear

1 A Lie algebra is locally finite if every finite set of elements is contained in a finite dimensional subalgebra.

${ }^{2}$ Since the enveloping algebra in this case is algebraic, this assumption is equivalent to the assumption that the enveloping algebra is semi-simple in the sense of Jacobson [9].

3 This Lemma is essentially due to Jacobson ([11]). 
combinations of the standard monomials $Y_{1}^{e_{1}} \cdots Y_{q}^{e_{q}}, e_{i} \geq 0$. On the other hand each 1.t. $Y_{i}$ satisfies a polynomial equation, so that at most a finite number of the standard monomials are linearly independent. Thus $\mathfrak{F}$, and in particular the subalgebra generated by the $A_{i}$, is finite dimensional. We leave the proof of the converse to the reader.

Proof of Theorem 3.1. First we prove that every element $X$ of $[\mathfrak{R}, \mathbb{R}] \cap \mathbb{C}$ is nilpotent. This fact can be proved by making use of a result of Chevalley ([2, p. 97]). The following direct proof was communicated to the author by Professor N. Jacobson. Let $X=\Sigma\left[X_{i} Y_{i}\right]$, where $X_{i}, Y_{i} \in \mathbb{R} ;$ then $\left[X X_{i}\right]=0$ for each $i$. The Lie subalgebra of $\mathfrak{Q}$ generated by $X, X_{1}, X_{2}, \cdots ; Y_{1}, Y_{2}, \cdots$ is finite dimensional, and by Lemma 3.1, its enveloping algebra $\&$ is a finite dimensional associative algebra. For each positive integer $k$, we have for all $i$,

$$
\left[X_{i} Y_{i}\right] X^{k}=X_{i} Y_{i} X^{k}-Y_{i} X_{i} X^{k}=\left[X_{i}, Y_{i} X^{k}\right] \text {. }
$$

Hence for each $k$,

$$
X^{k}=\sum\left[X_{i}, Y_{i} X^{k-1}\right],
$$

and it follows that the trace of $X^{k}$ in the regular representation of 5 is zero. This implies, since the characteristic of the base field is zero, that $X$ is nilpotent. But $X$ is in the center of the enveloping algebra $\mathfrak{A}$ of $\mathfrak{R}$, and hence the ideal $K X+X \mathfrak{H}$ is a nil two-sided ideal in $\mathfrak{A}$. Therefore $X=0$, and $[\Omega R] \cap \mathfrak{S}=0$.

Now let $\mathbb{R}_{1}$ be a subspace of $\mathfrak{Q}$ containing $[\mathfrak{R}, \mathfrak{R}]$, and complementary to $\mathfrak{C}$; then $\mathfrak{\Omega}_{1}$ is automatically an ideal, and $\mathbb{R}=\mathfrak{R}_{1} \oplus \mathfrak{C}$. We prove next that $\mathbb{R}_{1}$ has no nonzero solvable ideals.

As in [2], we observe that it is sufficient to prove that if $\mathbb{E}_{1}$ is an ideal in $\mathbb{R}$ containing $\mathbb{E}$ such that $\left[\mathbb{E}_{1}, \mathbb{E}_{1}\right] \leqq \mathbb{E}$ then $\mathbb{E}_{1} \leqq \mathbb{E}$. Since $\left[\mathfrak{C}_{1}, \mathbb{C}_{1}\right] \leqq \mathbb{S} \cap[\mathbb{R}, \mathfrak{R}]$, we have $\left[\mathfrak{C}_{1}, \mathbb{C}_{1}\right]=(0)$. Consider the ideal $\mathfrak{U}=\left[\mathfrak{C}_{1}, \mathfrak{l}\right]$; by the argument used in the first part of the proof it follows that every element in $\mathfrak{U}$ is nilpotent. Let $\mathfrak{B}$ be the enveloping algebra of $\mathfrak{u}$. Since $\mathfrak{U}$ is commutative, $\mathfrak{B}$ is a nil algebra. By a result of Jacobson [7, Lemma 1], the proof of which is applicable to the present situation, $\mathfrak{B O}$ is a two-sided ideal in the enveloping algebra $\mathfrak{A}$ of $\mathfrak{\Omega}$. We assert that $\mathfrak{B} \mathfrak{A}$ is a nil ideal. In fact, if $X=\sum B_{i} A_{i} \in \mathfrak{B} \mathfrak{A}, B_{i} \in \mathfrak{B}$, $A_{i} \in \mathfrak{A}$, then there exist elements $W_{1}, \cdots, W_{r}$ in $\mathfrak{U}$ such that the $B_{i}$ are in the enveloping algebra of the Lie subalgebra of $\mathfrak{U}$ generated by the $W_{i}$, and elements $X_{1}, \cdots, X_{s}$ in $\&$ such that the $A_{i}$ are in the enveloping algebra of the Lie subalgebra of $\mathbb{R}$ generated by the $X_{i}$, Since $\mathfrak{R}$ is locally finite, there exists a finite dimensional subalgebra $\Omega_{1}$ of $\mathfrak{L}$ containing $\left\{W_{1}, \cdots, W_{r} ; X_{1}, \cdots, X_{s}\right\} \ldots$ Let $\mathfrak{U}_{1}=\mathfrak{R}_{1} \cap \mathfrak{l}$; then $\mathfrak{u}_{1}$ is an ideal in $\mathfrak{\Omega}_{1}$ containing the $W_{i}$, and consisting of nilpotent l.t. Let $\mathfrak{B}_{1}$ and $\mathfrak{A}_{1}$ 
be the enveloping algebras of $\mathfrak{U}_{1}$ and $\mathfrak{\Omega}_{1}$ respectively; by Lemma 3.1 , these algebras are finite dimensional, and hence $\mathfrak{B}_{1}$ is a nilpotent subalgebra of $\mathfrak{H}_{1}$. From the way $\mathfrak{B}_{1}$ and $\mathfrak{H}_{1}$ were chosen, $X \in \mathfrak{B}_{1} \mathfrak{A}_{1}$. By [7, Lemma 1] again, $\mathfrak{B}_{1} \mathfrak{2}_{1}$ is a two-sided ideal in $\mathfrak{U}_{1}$, and nilpotent since $\mathfrak{B}_{1}$ is nilpotent. Then $X$ is nilpotent, $\mathfrak{B} \mathscr{U}$ is a nil ideal, and hence $\mathfrak{B} \mathfrak{U}=0$. Then $\mathfrak{B}$ itself is a nil ideal in $\mathfrak{A}$, so that $\mathfrak{B}=0$, and $\mathfrak{l}=\left[\mathfrak{C}_{1}, \mathfrak{Z}\right]$ $=0$, proving that $\mathfrak{L}_{1}$ contains no nonzero solvable ideals.

Finally let $X$ be an arbitrary 1.t. in 5 . Let $N$ be the nilpotent component of $X$; since $N$ is a polynomial in $X, N$ is in the center of the enveloping algebra $\mathfrak{A}$ of $\mathbb{R}$, and consequently is contained in a nil two-sided ideal in $\mathfrak{U}$, so that $N=0$. The theorem is now completely proved.

Corollary. A completely reducible Lie algebra \& of finite valued ${ }^{ \pm}$ l.t. in a vector space $\mathfrak{M}$ over a field $K$ of characteristic zero has the structure described in Theorem 3.1.

Proof. Our hypothesis means that $\mathfrak{M}$ is a completely reducible R-module; it follows that $\mathfrak{M}$ is also a completely reducible $\mathfrak{U}$-module, where $\mathfrak{A}$ is the enveloping algebra of $\mathfrak{L}$. The algebra $\mathfrak{U}$ contains no nonzero nil ideals because such an ideal is contained in the Jacobson radical of $\mathfrak{A}$, and the radical annihilates every completely reducible $\mathfrak{H}$-module. By [8, p. 243], every associative algebra of finite valued l.t. is locally finite. Hence $\&$ is locally finite, and we have shown that \& satisfies the hypotheses of Theorem 3.1.

EXAMPLE. We present an example of an algebra satisfying the hypotheses of Theorem 3.1, with the property that every subalgebra $\mathfrak{\Omega}_{1}$, complementary to $\mathfrak{5}$, properly contains [82]. Let $\mathfrak{M}$ be a vector space of countable dimension over $K$, and let $\&$ be the Lie algebra under $[X Y]=X Y-X Y$, of all l.t. of the form $\alpha 1+F$, where $\alpha \in K, 1$ is the identity l.t., and $F$ is a l.t. having a corner finite ${ }^{5}$ matrix relative to a fixed basis $\left\{u_{i}\right\}$ of $\mathfrak{M}$. The enveloping algebra $\mathfrak{A}$ of $\mathfrak{Z}$ is $\mathfrak{Z}$ itself, which is a dense algebra of l.t. in $\mathfrak{M}$, and consequently contains no nonzero nil ideals. Let $\mathfrak{S}$ be the subalgebra of $\mathfrak{\&}$ consisting of all l.t. in $\mathcal{Q}$ for which the coefficient of 1 is zero. Then $\mathfrak{H}$ is locally finite by [8], and since $\mathfrak{Q}$ has a basis consisting of a basis for $\mathfrak{H}$ and the identify l.t., $\mathfrak{Z}$ is locally finite. Clearly $[\mathbb{Q}, \mathfrak{R}]=[\mathfrak{S}, \mathfrak{S}]$, and thus, since $\mathfrak{F}$ is isomorphic to the Lie algebra of all corner finite matrices $X$, for which $\operatorname{tr} X$ has a meaning, [R, 2$]$ can be characterized as the algebra of all

${ }^{4}$ A 1.t. is finite valued if its range space is finite dimensional.

5 A 1.t. $A$ is corner finite relative to a basis $u_{1}, u_{2}, \cdots$ if for some positive integer $m,\left(u_{1}, \cdots, u_{m}\right) A \leqq\left(u_{1}, \cdots, u_{m}\right) ; u_{r} A=0, r>m$. 
corner finite matrices of trace zero. On the other hand, the center (5)

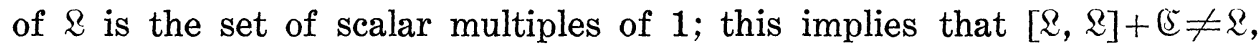
since there exist, in fact, infinitely many linearly independent l.t., namely the $E_{i}, u_{i} E_{j}=\delta_{j i} u_{i}$, such that for each $i, E_{i}-\alpha 1-F$ is not finite valued if $\alpha \neq 0$, and has a nonzero trace if $\alpha=0, F \in[\Omega, \Omega]$.

It follows from Theorem 3.1 that $\mathfrak{S}$ has no nonzero solvable ideals; nevertheless we have shown that $[\mathfrak{S}, \mathfrak{S}] \neq \mathfrak{K}$, proving that in general Lie algebras of finite valued l.t. containing no nonzero solvable ideals are not direct sums of simple Lie algebras.

The following application of Theorem 3.1 to abstract Lie algebras is motivated by Koszul's characterization [12, p. 87] in the finite dimensional case, of reductive Lie algebras. that is, Lie algebras $\&$ which are completely reducible relative to ad?.

THEOREM 3.2. Let 2 be a Lie algebra over an arbitrary field $K$ of characteristic zero. If ad\& is a completely reducible, locally finite algebra of algebraic l.t. then there exists an ideal $\mathfrak{L}_{1}$ containing [R, 2$]$ and possessing no nonzero solvable ideals such that $\mathbb{Q}=\mathfrak{Q}_{1} \oplus \mathfrak{C}$, where $\mathfrak{E}$ is the center of $\mathrm{R}$.

Proof. Since $\mathbb{Q}$ is a completely reducible ad $\mathbb{Q}$-module, there exists an ideal $\mathfrak{R}_{1}$ such that $\mathbb{L}=\mathbb{R}_{1} \oplus \mathbb{C}$. Then $\mathfrak{Q}_{1} \cong \mathbb{L} / \mathbb{C} \cong a d \mathbb{R}$. The argument used in the proof of the Corollary to Theorem 3.1 implies that the enveloping algebra of ad\& contains no nonzero nil ideals, and therefore, by Theorem 3.1 , adR, and hence $\mathfrak{L}_{1}$, is a direct sum $\mathfrak{R}_{1}=\mathbb{R}_{1}^{*}+\mathfrak{r}_{1}$ where $\Omega_{1}^{*}$ contains $\left[\Omega_{1}, \Omega_{1}\right]=[\Omega, \Omega]$ and possesses no nonzero solvable ideals, and $\mathfrak{C}_{1}$ is the center of $\mathfrak{Q}_{1}$. But since $\mathbb{R}=\mathbb{R}_{1}^{*} \oplus \mathbb{E}_{1} \oplus \mathfrak{C},\left[\mathfrak{C}_{1}, \mathbb{R}\right]=0, \mathbb{E}_{1} \leqq \mathfrak{C}$; thus $\mathfrak{C}_{1}=0, \Omega_{1}^{*}=\mathbb{Q}_{1}$, and the theorem is proved.

\section{An application to solvable Lie algebras.}

THeOREM 4.1. Let 2 be a solvable Lie algebra over an arbitrary field $K$ with the property that for every $x \in \mathfrak{R}$, adx is an algebraic l.t.. Then $\&$ is locally finite.

Proof. (Cf. [13, pp. 181, 182]) Since $\mathbb{Z}$ is solvable, $\mathbb{Z}$ has a finite normal series whose factors are commutative:

$$
0=\mathbb{R}_{0} \subset \mathbb{R}_{1} \subset \cdots \subset \mathbb{R}_{r}=\mathrm{R} \text {, and }\left[\mathbb{R}_{i}, \mathbb{R}_{i}\right] \leqq \mathbb{R}_{i-1} .
$$

Clearly $\mathfrak{Q}_{0}$ is locally finite. Then, by an induction on $r$, we may assume that $\mathbb{R}_{r-1}$ is locally finite. Evidently we may apply Zorn's lemma to the inclusion ordered set of locally finite subalgebras of $\&$ containing $\mathfrak{Q}_{r-1}$; let $\mathbb{F}$ be a maximal element in this set. Since $[\mathbb{R}, \mathbb{R}] \leqq \mathfrak{Q}_{r-1}$, $\mathbb{F}$ is 
an ideal in 2. Let $x$ be an arbitrary element of $\mathrm{R}$; then we shall prove that the subalgebra $\mathfrak{U}$ generated by $\mathbb{F}$ and $x$ is locally finite, and from this, in view of the maximality of $\mathbb{r}$, we shall conclude that $\mathbb{L}=\mathbb{F}$. Since $[\mathfrak{S}, x] \leqq \mathfrak{\Im}$, it follows that $\mathfrak{l l}$ coincides with the subspace generated by $\left(\xi\right.$ and $x$. Let $u_{1}, \cdots, u_{s}$ be a finite set of elements of $\mathfrak{u}$; then each $u_{i}$ can be expressed in the form $u_{i}=e_{i}+\alpha_{i} x, e_{i} \in \mathbb{F}, \alpha_{i} \in K$. Since $a d x$ is an algebraic l.t., the $e_{i}$ are contained in a finite dimensional subspace $\mathfrak{X}$ of $\mathfrak{F}$ such that $[\mathfrak{X}, x] \leqq \mathfrak{X}$. If $\mathfrak{Y}$ is the subalgebra of $\mathfrak{F}$ generated by the elements of $\mathfrak{X}$, then because the elements of $\mathfrak{X}$ are linear combinations of multiple commutators involving the elements of $\mathfrak{X}$, it follows from the Jacobi identity that $[\mathfrak{Y}, x] \leqq \mathfrak{Y}$. On the other hand, $\mathfrak{Y}$ is finite dimensional since $\mathfrak{X} \leqq \mathbb{F}$. Therefore all the $u_{i}$ are contained in the finite dimensional subalgebra $\mathfrak{Y}+(x)$, and the proof is complete.

COROLlaRY. Let $\mathbb{2}$ be a solvable Lie algebra of l.t. in a vector space $\mathfrak{M}$ over $K$ such that every l.t. in $\mathbb{Q}$ is algebraic. Then $\mathbb{L}$ is locally finite.

Proof. By Theorem 2.3, if $X \in \mathcal{Q}$, then $a d X$ is an algebraic l.t., and we can apply Theorem 4.1.

Theorem 4.2. Let 2 be a solvable Lie algebra over a field $K$ of characteristic zero consisting of algebraic l.t., and assume that the enveloping algebra of $\mathfrak{Q}$ is semi-simple. Then $[\mathfrak{R}, \mathfrak{Q}]=0$.

Proof. By the Corollary to Theorem $4.1, \mathbb{2}$ satisfies the hypotheses of Theorem 3.1 , and therefore there exists a subalgebra $\mathfrak{L}_{1}$ of $\mathfrak{R}$ containing $[\&, \&]$ and possessing no nonzero solvable ideals, such that $\mathbb{R}=\mathbb{R}_{1} \oplus \mathbb{C}$, where $\mathbb{C}$ is the center of $\mathbb{R}$. Then $[\mathbb{R}, \mathbb{R}]$ is a solvable ideal in $\mathfrak{Q}_{1}$, and hence $[\mathfrak{Q}, \mathfrak{Q}]=0$.

CoROLlary. Let $x \rightarrow U_{x}$ be an irreducible representation of a solvable Lie algebra 2 over an algebraically closed field $K$ of characteristic zero, such that for every $x \in \mathbb{R}, U_{x}$ is an algebraic l.t.. Then the representation space is one dimensional.

Proof. The Lie algebra $\mathscr{Q}_{1}$ consisting of the $U_{x}$ is locally finite and hence the enveloping algebra $\mathfrak{A}$ of $\mathfrak{Z}_{1}$ is algebraic, by Lemma 3.1. Since $\mathfrak{A}$ is an irreducible algebra of 1.t., $\mathfrak{A}$ contains no nonzero nil ideals, and hence, by Theorem 4.2, $\mathfrak{U}$ is commutative, By Schur's Lemma, the nonzero elements of $\mathfrak{A}$ are automorphisms of the representation space, and because they are algebraic, and $K$ is algebraically closed, 
they are scalar multiplications. Thus the dimension of the representation space is equal to one.

As a further applicated of Theorem 4.1, we give a new proof of a result of Gruenberg ${ }^{6}$.

THEOREM 4.3. (Gruenberg) Let $\mathbb{2}$ be a finitely generated solvable Lie algebra over an arbitrary field $K$ such that for every $x \in \mathbb{R}$, adx is a nilpotent l.t. Then $\mathbb{2}$ is a nilpotent Lie algebra.

Proof. Since $x \rightarrow a d x$ is a homomorphism of $\mathfrak{L}$ onto $a d \mathbb{R}$, ad\& is a finitely generated solvable algebra of nilpotent 1.t. By Theorem 4.1, $a d \mathbb{R}$ is finite dimensional, and hence, by a result of Jacobson [10], the enveloping algebra $\tilde{a}$ of $a d \mathbb{R}$ is a nilpotent associative algebra. If $\mathrm{C}^{m}=0$, then it follows directly that $\mathrm{R}^{m+1}=0$,

In the finite dimensional case, Theorem 4.2 can be applied to prove that if $\mathbb{R}$ is a solvable algebra of matrices with coefficients in an algebraically closed field of characteristic zero, then all the matrices in $\&$ can be put simultaneously in triangular form. Another well known result [3] states that if the field is algebraically closed and if $\&$ is a commutative set of semi-simple l.t., then the vector space has a basis consisting of vectors which are proper vectors for all the l.t. in 2. Neither result holds in the infinite dimensional case. In fact, we shall construct a commutative associative algebra $\&$ of semi-simple algebraic l.t. in a vector space $\mathfrak{M}$ of countable dimension over an arbitrary field $K$ with the property that not every vector in $\mathfrak{M}$ is contained in a finite dimensional \&-submodule. Obviously the matrices belonging to such a set of 1.t. cannot be put in triangular form, and, a fortiori, not in diagonal form.

Let $\mathfrak{M}$ be a vector space with basis element $e_{1}, e_{2}, \cdots$. $\mathfrak{M}$ becomes a commutative associative algebra over $K$ if we define multiplication of the basis elements by

$$
e_{i}^{2}=e_{i}, \quad e_{i} e_{j}=e_{k}, \quad k=\max (i, j),
$$

if $i \neq j$.

Let $\mathbb{R}$ be the commutative associative algebra of multiplications $R_{x}$ : $a \rightarrow a x, a$ and $x$ in $\mathfrak{M}$. Then it follows from (1) that every 1.t. in $\mathbb{R}$ is algebraic. Moreover, since $x \rightarrow R_{x}$ is a homomorphism, the minimum polynomial of each $R_{e_{i}}$ divides the polynomial $t^{2}-t$, from which it follows easily that the $R_{e_{i}}$ are semi-simple 1.t.. For each $x$ in $\mathfrak{M}, R_{x}$ is a linear combination of a finite number $R_{e_{1}}, \cdots, R_{e_{s}}$ of the generators. Let $a \in \mathfrak{M}$; then since the $R_{e_{i}}$ commute, $a$ is contained in a finite dimensional space $\mathfrak{N}$ invariant relative to the $R_{e_{i}}$. The restriction of the $R_{e_{i}}$

${ }^{6}$ See [6]. Gruenberg treats Lie rings with coefficients in an arbitrary commutative ring instead of Lie algebras over a field. 
to $\mathfrak{N}$ are semi-simple, therefore we infer (see [3, p. 69]) that the restriction of $R_{x}$ to $\mathfrak{R}$ is semi-simple. Thus every element of $\mathfrak{M}_{i}$ is contained in a sum of irreducible $R_{x}$-modules, and hence $R_{x}$ is semisimple. Finally, because of (1), the vector $e_{1}$, for example, is not contained in any finite dimensional $\mathbb{Q}$-submodule of $\mathfrak{M}$.

5. Linearly splittable algebras of 1.t. A Lie algebra $\&$ of algebraic 1.t. over an arbitrary field of characteristic zero is said to be linearly splittable ( $l$-splittable) if for each $X$ in $\mathfrak{R}$, the semi-simple and nilpotent components of $X$ are also elements of $\mathbb{2}$. It is immediate that the intersection of any number of $l$-splittable algebras is $l$-splittable. Because the semi-simple and nilpotent components of a l.t. are polynomials in the l.t., any associative algebraic algebra of l.t., viewed as a Lie algebra, is $l$-splittable. Therefore every locally finite Lie algebra $\mathfrak{R}$ of l.t. is contained in a uniquely determined minimal $l$-splittable Lie algebra $2^{*}$, namely the intersection of all the $l$-splittable algebras containing it. We have only to check that $\mathfrak{Q}$ is contained in at least one $l$-splittable algebra; the enveloping algebra is the obvious candidate, and it is algebraic by Lemma 3.1. We shall call $\mathfrak{Q}^{*}$ the splitting of $\mathfrak{R}$. It is not difficult to prove that the properties of the algebraic hull of a linear Lie algebra stated in Prop. $1, \S I$, of [2], also hold for the splitting of a locally finite Lie algebra of l.t..

LEMMA 5.1. Let $\mathfrak{R}$ be a solvable algebra of algebraic l.t. in a vector space $\mathfrak{M}$ over an arbitrary field $K$ of characteristic zero. Then the nilpotent l.t. in $\&$ form an ideal $\mathfrak{R}$ containing [R\&].

Proof. Theorem 4.1, together with Lie's theorem [7, p. 879] in the finite dimensional case, imply that every element of [22] is nilpotent. Now let $X$ and $Y$ be arbitrary nilpotent l.t. in 2 . Then $X+Y$ is algebraic, and since every element of $\mathfrak{M}$ is contained in a finite dimensional subspace invariant under $X+Y, X+Y$ is nilpotent if we can prove that its restriction to every finite dimensional $(X+Y)$-module is nilpotent. Let $\mathfrak{F}$ be such a submodule; let $F_{1}$ be the enveloping algebra of the solvable algebra $\mathfrak{Q}_{1}$ generated by the restrictions $X_{F}$ and $Y_{F}$ of $X$ and $Y$ to $\mathfrak{F}$; and let $\mathfrak{U}_{1}$ be the enveloping algebra of $\left[\mathfrak{R}_{1}, \mathfrak{R}_{1}\right]$. Then $\mathfrak{U}_{1} \mathfrak{F}_{1}$ is a two-sided ideal in $\mathfrak{F}_{1}$ by $[7$, Lemma 1$]$, and a nilpotent ideal because $\mathfrak{U}_{1}$ is a nilpotent algebra by the remark at the beginning of the proof, and Engel's theorem. Since $X$ and $Y$ are nilpotent, it follows that some power of $(X+Y)_{F}$ is in $\mathfrak{U}_{1} \mathfrak{\xi}_{1}$, and hence $X+Y$ is nilpotent. Thus the nilpotent elements form a subspace of $\mathfrak{R}$, and hence an ideal containing [RQ].

Following Malcev [14], we call $\mathfrak{R}$ the kernel of $\mathfrak{R}$. 
TheOREM 5.1. Let $\mathbb{Q}$ be a solvable Lie algebra of countable dimension over an arbitrary field of characteristic zero, all of whose elements are algebraic l.t. in a vector space $\mathfrak{M}$. If $\mathbb{L}$ is l-splittable, then there exists a commutative subalgebra $\mathfrak{B}$ of $\mathbb{R}$ consisting of semi-simple l.t. such that $\mathfrak{R}=\mathfrak{B}+\mathfrak{R}, \mathfrak{B} \cap \mathfrak{R}=0$, where $\mathfrak{R}$ is the kernel of $\mathfrak{R}$.

Proof. First we observe that any finite dimensional subalgebra $\mathfrak{S}$ of $\mathfrak{Z}$ is contained in a finite dimensional $l$-splittable subalgebra of $\mathbb{R}$, namely the intersection of $\mathbb{L}$ with the enveloping algebra of $\mathfrak{S}$. From this, the local finiteness, and the fact that $\&$ has countable dimension over $K$, it follows that there is a sequence

$$
\Omega_{1} \leqq \Omega_{2} \subseteq \cdots
$$

of $l$-splittable, finite dimensional subalgebras of $\&$ whose union is $\&$. If $\mathfrak{R}_{i}$ is the kernel of $\mathfrak{R}_{i}$, then $\mathfrak{N}_{i}=\mathfrak{N} \cap \mathfrak{R}_{i}$.

We make two preliminary observations. (1) Any commutative set of semi-simple 1.t. in $\mathfrak{R}_{i}, i=1,2, \cdots$ is contained in a maximal commutative subalgebra of $\mathfrak{Q}_{i}$ consisting of semi-simple l.t. (2) If $\mathfrak{B}_{i}$ is any maximal commutative subalgebra of $\mathfrak{R}_{i}$ consisting of semi-simple 1.t., then $\mathfrak{Q}_{i}=\mathfrak{B}_{i}+\mathfrak{N}_{i}, \mathfrak{B}_{i} \cap \mathfrak{R}_{i}=0$. For the proof of (2) we see that by Theorem 2.3, $a d_{L_{i}} \mathfrak{B}_{i}$ is a commutative finite dimensional set of semisimple l.t. acting in $\mathfrak{R}_{i}$, and hence $\mathfrak{R}_{i}$ is a completely reducible $\alpha d_{L_{i}} \mathfrak{B}_{i^{-}}$ module. If $\mathfrak{X}_{0}=\left\{X \mid X \in \mathbb{R}_{1},\left[X \mathfrak{B}_{i}\right]=0\right\}$, then $\mathfrak{X}_{0}$ is a submodule, and hence there is a submodule $\mathfrak{X}_{1}$ such that $\mathfrak{R}_{i}=\mathfrak{X}_{0} \oplus \mathfrak{X}_{1}$. If $\mathfrak{X}$ is an irreducible submodule contained in $\mathfrak{X}_{1}$, then $\left[\mathfrak{X P}_{i}\right] \neq 0$, and hence $\left[\mathfrak{X}_{i}\right]=\mathfrak{X}$. It follows that $\mathfrak{X}_{1} \subseteq\left[\mathcal{Q}_{i} \mathbb{Q}_{i}\right] \subseteq \mathfrak{N}_{i}$. On the other hand, if $X \in \mathfrak{X}_{0}$ then $X=S+N$, where $S$ and $N$ are the semi-simple and nilpotent components of $X$. Since $\mathfrak{R}_{i}$ is $l$-splittable, $N \in \mathfrak{N}_{i}$, and $S$, which is a polynomial in $X$, commutes with every element of $\mathfrak{B}_{i}$. But $\mathfrak{B}_{i}$ is a maximal commutative algebra of semi-simple 1.t., hence $S \in \mathfrak{B}_{i}$, and $\mathfrak{X}_{0} \subseteq \mathfrak{B}_{i}+\mathfrak{R}_{i}$. Thus $\mathfrak{R}_{i}=\mathfrak{B}_{i}$ $+\mathfrak{N}_{i}$. Finally $\mathfrak{B}_{i} \cap \mathfrak{N}_{i}=0$, since a l.t. which is both semi-simple and nilpotent is the zero l.t..

We now construct $\mathfrak{B}$ by induction, beginning with a maximal commutative subalgebra $\mathfrak{B}_{1}$ of $\mathfrak{Z}_{1}$ consisting of semi-simple l.t.. If at some stage we have constructed $\mathfrak{B}_{1} \subseteq \mathfrak{B}_{2} \subseteq \cdots \subseteq \mathfrak{B}_{k}$ such that each $\mathfrak{B}_{j} \subseteq \mathbb{R}_{j}$, $\mathfrak{R}_{j}=\mathfrak{B}_{j} \oplus \mathfrak{R}_{j}$, where $\mathfrak{B}_{j}$ is a commutative subalgebra consisting of semisimple l.t., then by (1) and (2), $\mathfrak{B}_{k}$ is contained in a maximal commutative subalgebra $\mathfrak{B}_{k+1}$ of $\mathfrak{R}_{k+1}$ with the same properties. Let $\mathfrak{B}=\bigcup_{k=1}^{\infty} \mathfrak{B}_{k}$; then $\mathfrak{B}$ is a commutative subalgebra of $\mathbb{Q}$ consisting of semi-simple l.t., and $\mathfrak{B} \cap \mathfrak{R}=0$. If $X \in \mathbb{R}$, then $X \in \mathbb{R}_{k}$ for some $k$, and we see that $X=B+N, B \in \mathfrak{B}, N \in \Re$.

A natural generalization of solvability is the concept of $\omega$-solvability, 
an algebra $\&$ being $\omega$-solvable if the intersection of the terms in the derived series of $\mathfrak{Z}$ is the zero space. For example, the algebra of all corner finite triangular matrices on a countably infinite index set is locally finite, $l$-splittable, and $\omega$-solvable, but not solvable.

THEOREM 5.2. Let $\&$ be an l-splittable, locally finite w-solvable algebra of algebraic l.t., and assume that 2 has countable dimension over $K$. Then the nilpotent l.t. in $\mathfrak{Q}$ form an ideal $\mathfrak{R}$ containing [, $\mathfrak{Q}]$, and there exists a commutative subalgebra $\mathfrak{B}$ of $\mathbb{Q}$ consisting of semi-simple l.t. such that $\mathfrak{Q}=\mathfrak{B}+\mathfrak{R}, \mathfrak{B} \cap \mathfrak{N}=0$.

Proof. We have only to remark that Lemma 5.1 in the countable case, and the proof of Theorem 5.1 required only that $\&$ be locally finite, and the union of an increasing sequence of finite dimensional solvable subalgebras. These conditions are guaranteed by the hypotheses of the theorem, since a finite dimensional $\omega$-solvable algebra is solvable.

TheOrem 5.3. Let $\&$ be a solvable Lie algebra of semi-simple algebraic l.t. of countable dimension over $K$. Then 2 is commutative.

Proof. The hypothesis of the theorem implies that $\&$ is $l$-splittable, and that the kernel of $\&$ is the zero space. The conclusion follows from Theorem 5.1.

REMARKS AND EXAMPLES. We cannot assert in general that the kernel $\mathfrak{R}$ of a solvable algebra of algebraic l.t. has a structure much more transparent than that of the original algebra. It is easy to show that $\mathfrak{N}$ is locally nilpotent in the sense that every finite subset of $\mathfrak{N}$ generates a nilpotent subalgebra. But $\mathfrak{R}$ is not necessarily nilpotent. We shall give an example of an algebra $\mathfrak{Q}$ such that every element of ad $\mathbb{R}$ is nilpotent, $\mathbb{R}$ is solvable, but $\mathfrak{Z}$ is not nilpotent. The example we construct has the property that $[\mathbb{Q}, \mathfrak{Q}]$ is commutative. Let $\mathfrak{Q}$ be a commutative algebra, and let $D$ be a commutative algebra of nilpotent derivations acting in $\mathbb{R}$, containing derivations of arbitrarily high index of nilpotency. Such an $\mathcal{L}$ and $D$ are easy to construct, and we omit the details. Then the semi-direct sum $\mathfrak{L}+\mathfrak{D}$ of $\mathfrak{L}$ and $\mathfrak{D}$ has the required properties.

We shall use an example due to Zelinsky [15] to show that the conclusion of Theorem 5.3 is false if no countability hypotheses are assumed. Zelinsky has constructed an associative algebra $\mathfrak{M}$ over an arbitrary field $K$, with radical $\Re$ such that $\mathfrak{R}^{2}=0, \mathfrak{M} / \mathfrak{R}$ is commutative, $\mathfrak{M}$ is locally finite, and such that $\mathfrak{M}$ contains no subalgebra $\mathfrak{B}$ mapped canonically upon $\mathfrak{M} / \mathfrak{R}$ unless $\mathfrak{M}$ has countable dimension over $K$. $\mathfrak{M}$ 
has a basis $\left\{e_{i}, n_{i j} \mid i, j \in I\right\}$ and multiplication table

$$
\begin{array}{ll}
e_{i}^{2}=e_{i}, \quad e_{i} e_{j}=n_{i j}, i \neq j, & e_{i} n_{j k}=\delta_{i j} n_{j k}, \\
n_{i k} e_{i}=\delta_{k i} n_{j k}, \quad n_{i j} n_{k 1}=0 & \text { (Kronecker deltas.) }
\end{array}
$$

Let $\mathfrak{Q}$ be the associative algebra of right multiplications $R_{s}, s \in \mathfrak{M}$, viewed as a Lie algebra under commutation. Then $\mathfrak{L}$ is a locally finite associative algebra, and hence is an $l$-splittable algebra of algebraic l.t.. From the table we see that if $x, y \in \mathfrak{M}$, then $[x y] \in \Re$, hence $\left[[x y],\left[x^{\prime} y^{\prime}\right]\right]$ vanishes identically, so that $\mathbb{R}$ is solvable. We notice that $R_{x}$ is nilpotent if and only if $x \in \mathfrak{R}$; thus the kernel $\mathfrak{R}$ of $\mathbb{R}$ consists of all $R_{x}, x \in \mathfrak{R}$. Suppose that $\mathfrak{Z}=\mathfrak{B}+\mathfrak{R}, \mathfrak{B} \cap \mathfrak{i}=0$, where $\mathfrak{B}$ is a commutative Lie algebra consisting of semi-simple 1.t.. The enveloping associative algebra $\mathfrak{C}$ of $\mathfrak{B}$ is a commutative subalgebra of $\mathbb{Z}$ consisting of semi-simple l.t., by an argument similar to that used in the example of $\S 4$. Therefore $\subseteq \cap \mathfrak{N}=0$. On the other hand, it can be verified directly that $x \rightarrow R_{x}$ is an isomorphism of $\mathfrak{M}$ onto $\mathfrak{L}$, so that if $\mathfrak{D}$ is the inverse image of $\mathfrak{C}$ under $x \rightarrow R_{x}$, we have $\mathfrak{i}=\mathfrak{D}+\mathfrak{i}, \mathfrak{D} \cap \mathfrak{l}=0$. Zelinsky has shown that this implies that the index set $I$ is denumerable, and we conclude that if $I$ is non-denumerable, then $\&$ cannot have a decomposition according to Theorem 5.3.

\section{REFERENCES}

1. C. Chevalley, $A$ new kind of relationship between matrices, Amer. J. Math., 6 (1943), 521-531.

2. . . Algebraic Lie algebras, Annals of Math., 48 (1947), 91-100.

3. . . Theorie des Groupes de Lie II (Groupes Algebriques) Paris, 1951.

4. M. Drazin and K. Gruenberg, Commutators in associative rings, Proc. Cambridge Philos. Soc., 49 (1953), 590-594.

5. M. Drazin, Triangular representations of linear algebras, Proc. Cambridge Philos. Soc., 49 (1953), 595-600.

6. K. Gruenberg, Two theorems on Engel groups, Proc. Cambridge Philos. Soc., 49 (1953), 377-380.

7. N. Jacobson, Rational methods in the theory of Lie algebras, Annals of Math., 36 (1935), 875-881.

8. - _.... Structure theory of simple rings without finiteness assumptions, Trans. Amer. Math. Soc., 57 (1945), 228-245.

9. - The radical and semi-simplicity for arbitrary rings, Amer. J. Math., 67 (1945), 300-320.

10. Une généralisation du theoreme d'Engel, C. R. Acad. Sci. Paris, 234 (1952), 679-681.

11. _ Lie and Jordan triple systems, Amer. J. Math., 71 (1949), 160.

12. J. L. Koszul, Homologie et cohomologie des algebres de Lie, Bull. Soc. Math. France,

78 (1950), 65-127.

13. A. G. Kurosch, Gruppentheorie, Berlin, 1953.

14. A. I. Malcev, Solvable Lie algebras, Amer. Math. Soc. Translation no. 27. 
15. E. Schenkman, Infinite Lie algebras, Duke Math. J., 19 (1952), 529-535.

16. D. Zelinsky, Raising idempotents, Duke Math. J., 21 (1954), 315-322.

UNIVERSITY OF WISCONSIN AND

InSTITUTE FOR AdVANCED STUDY 


\section{PACIFIC JOURNAL OF MATHEMATICS}

\section{EDITORS}

\author{
H. L. Royden \\ Stanford University \\ Stanford, California \\ E. Hewitt \\ University of Washington \\ Seattle 5 , Washington
}

R. P. Dilworth

California Institute of Technology

Pasadena 4, California

\author{
A. HorN* \\ University of California \\ Los Angeles 24, California
}

\section{ASSOCIATE EDITORS}

\author{
E. F. BECKENBACH \\ C. E. BURGESS \\ H. BUSEMANN \\ H. FEDERER
}

\author{
M. HALL \\ P. R. HALMOS \\ V. GANAPATHY IYER \\ R. D. JAMES
}

\author{
M. S. KNEBELMAN \\ I. NIVEN \\ T. G. OSTROM \\ M. M. SCHIFFER
}

J. J. STOKER

G. SZEKERES

F. WOLF

K. YOSIDA

\section{SPONSORS}

\author{
UNIVERSITY OF BRITISH COLUMBIA \\ CALIFORNIA INSTITUTE OF TECHNOLOGY \\ UNIVERSITY OF CALIFORNIA, BERKELEY \\ UNIVERSITY OF CALIFORNIA, DAVIS \\ UNIVERSITY OF CALIFORNIA, LOS ANGELES \\ UNIVERSITY OF CALIFORNIA, SANTA BARBARA \\ MONTANA STATE UNIVERSITY \\ UNIVERSITY OF NEVADA \\ OREGON STATE COLLEGE \\ UNIVERSITY OF OREGON \\ UNIVERSITY OF SOUTHERN CALIFORNIA
}

STANFORD RESEARCH INSTITUTE

STANFORD UNIVERSITY

UNIVERSITY OF UTAH

WASHINGTON STATE COLLEGE

UNIVERSITY OF WASHINGTON

AMERICAN MATHEMATICAL SOCIETY HUGHES AIRCRAFT COMPANY SHELL DEVELOPMENT COMPANY

Mathematical papers intended for publication in the Pacific Journal of Mathematics should be typewritten (double spaced), and the author should keep a complete copy. Manuscripts may be sent to any of the editors. Manuscripts intended for the outgoing editors should be sent to their successors. All other communications to the editors should be addressed to the managing editor, Alfred Horn at the University of California, Los Angeles 24, California.

50 reprints of each article are furnished free of charge; additional copies may be obtained at cost in multiples of 50 .

The Pacific Journal of Mathematics is published quarterly, in March, June, September, and December. The price per volume (4 numbers) is $\$ 12.00$; single issues, $\$ 3.50$. Back numbers are available. Special price to individual faculty members of supporting institutions and to individual members of the American Mathematical Society: $\$ 4.00$ per volume; single issues, $\$ 1.25$.

Subscriptions, orders for back numbers, and changes of address should be sent to Pacific Journal of Mathematics, c/o University of California Press, Berkeley 4, California.

Printed at Kokusai Bunken Insatsusha (International Academic Printing Co., Ltd.), No. 10, 1-chome, Fujimi-cho, Chiyoda-ku, Tokyo, Japan.

* During the absence of E. G. Straus.

PUBLISHED BY PACIFIC JOURNAL OF MATHEMATICS, A NON-PROFIT CORPORATION COPYRIGHT 1956 BY PACIFIC JOURNAL OF MATHEMATICS 


\section{Pacific Journal of Mathematics}

\section{Vol. 6, No. $3 \quad$ BadMonth, 1956}

Richard Arens and James Eells, Jr., On embedding uniform and topological spaces ............................................ 397

N. Aronszajn and Prom Panitchpakdi, Extension of uniformly continuous transformations and hyperconvex metric spaces .............. 405

Kai Lai Chung and Cyrus Derman, Non-recurrent random walks ........ 441

Harry Herbert Corson, III, On some special systems of equations . . . . . . . . . 449

Charles W. Curtis, On Lie algebras of algebraic linear transformations . . . 453

Isidore Heller, Neighbor relations on the convex of cyclic permutations . . . . 467

Solomon Leader, Convergence topologies for measures and the existence of transition probabilities..................................... 479

D. H. Lehmer, On certain character matrices ...................... 491

Michael Bahir Maschler, Minimal domains and their Bergman kernel function ........................................... 501

Wm. M. Myers, Functionals associated with a continuous transformation ................................... 517

Irving Reiner and Jonathan Dean Swift, Congruence subgroups of matrix groups ....................................... 529

Andrew Sobczyk, Simple families of lines ................... 541

Charles Standish, A class of measure preserving transformations ........ 553 Jeremiah Milton Stark, On distortion in pseudo-conformal mapping ..... 565 